\title{
La «edad de oro» de las relaciones industriales: elementos de interpretación teórica ${ }^{1}$
}

\section{Miguel Ángel García Calavia}

Universitat de València. Departament de Sociologia i Antropologia Social Edifici Departamental Oriental. Avda. Tarongers, s/n. 46022 València Miguel.A.Garcia@uv.es

\section{Resumen}

En las décadas centrales del siglo XX se producen numerosos textos dedicados a sistematizar teóricamente el ámbito de estudio de las «relaciones industriales». En el presente artículo, se examinan algunas de las aproximaciones que van desde la perspectiva funcionalista hasta la teoría de la acción pasando por la marxista.

El fin que subyace a esta revisión parcial es poner de manifiesto cómo se configuran las relaciones laborales como dominio autónomo en un espacio geográfico, el del mundo anglosajón, y en un periodo determinado, el que se prolonga desde finales de la Segunda Guerra Mundial hasta la crisis de los setenta, destacando los conceptos bajo los que se constituye o los aspectos metodológicos que resultan más significativos para el estudio de los procesos de mediación institucional.

Palabras clave: teoría sociológica, relaciones industriales, institucionalismo.

\section{Resum. L'«edat d'or» de les relacions industrials: elements d'interpretació teòrica}

A les dècades centrals del segle XX es produeixen nombrosos textos dedicats a sistematitzar teòricament l'àmbit d'estudi de les «relacions industrials». En el present article, s'hi examinen algunes de les aproximacions que van des de la perspectiva funcionalista fins a la teoria de l'acció passant per la marxista.

El fi subjacent a aquesta revisió parcial és posar de manifest com es configuren les relacions laborals com a domini autònom en un espai geogràfic, el del món anglosaxó, i en un període determinat, el que es perllonga des de finals de la Segona Guerra Mundial fins a la crisi dels setanta, destacant els conceptes sota els quals es constitueix o els aspectes metodològics que resulten més significatius per a l'estudi dels processos de mediació institucional.

Paraules clau: teoria sociològica, relacions industrials, institucionalisme.

Abstract. "The golden age» of industrial relations: elements for a theoretical interpretation

In the middle of the twentieth century lots of researches devoted to systematize the field of study of «industrial relations». In this article some of the approaches - from the functio-

1. Quiero agradecer los comentarios efectuados a una primera versión de este artículo por Antonio Martín y Carlos Prieto. 
nalistic outloock to the theory of action and also considering the marxist view - are examined.

The final aim underlyng this revision is to show how labour relations are shaped as an autonomous domain on a certain geographical space - the anglo-saxon world- and for a certain period of time - from the end of Second World War to the crisis of the seventies-. We have underlined the concepts under which its is set up or the methodological aspects which turn out to be more meaningful for the study of the procedures of institutional mediation.

Key words: sociological theory, industrial relations, institutionalism.

\section{Sumario}

Los «primeros tiempos»: emergencia de una preocupación

El contexto intelectual

El prisma funcionalista de Dunlop: los sistemas de relaciones industriales

La perspectiva pluralista de la Escuela de Oxford: las instituciones de regulación laboral
Las perspectivas sistémicas a finales de los sesenta: primera crisis

El enfoque marxista: los procesos de control sobre las relaciones de empleo

Teoría de juegos y behaviorismo: la negociación colectiva

Un balance de la «edad de oro»

Bibliografía

Las relaciones laborales, transcripción peculiar del literal inglés «relaciones industriales» (industrial relations), en tanto que materia académica, es decir, como un objeto de estudio considerado dentro de una perspectiva científica, están delimitadas en la actualidad. Sin embargo, no ha sido siempre así. En el período comprendido entre las dos grandes guerras, su ámbito de estudio no está suficientemente perfilado, ni contextualizado sociológicamente. En el mundo anglosajón, más concretamente, en el Reino Unido, Sidney y Beatrice Webb, dos de los principales pioneros, ofrecen una mirada limitada a principios del siglo XX: ¿cómo los sindicatos con su acción contribuyen directamente a imponer principios democráticos en el centro de trabajo, para después intentar extenderlos al conjunto de la economía? En Estados Unidos, el ámbito de estudio es compartido por sociólogos, economistas o psicólogos, o mejor, por institucionalistas heterodoxos, neoclásicos o especialistas en relaciones humanas.

Algo similar sucede en el mundo centroeuropeo, muy convulsionado en los años veinte, donde Korsch centra su mirada en la lógica del desarrollo de la democracia industrial a partir de las distintas formas de implicación de los trabajadores en las decisiones relativas a las relaciones de empleo y a las organizaciones del conjunto de la economía.

Caire (1991) considera que el despegue propiamente dicho de este ámbito de estudio, lo que denomina la «edad de oro», se abre en 1933 con la National Industrial Recovery Act (NIRA), que normaliza las relaciones laborales en los Estados Unidos reconociendo el derecho de los asalariados a la negociación y 
a la organización sindical; ahora bien, será en las décadas posteriores a la Segunda Guerra Mundial cuando se desarrolle como disciplina. Frente a la visión de Elton Mayo que «percibe la fábrica y la oficina como la versión moderna de la tribu y al directivo como el jefe tribal de la comunidad», Clark Kerr señala, en un balance retrospectivo, que después de los años cuarenta se efectúa «un esfuerzo para releer de manera más realista la teoría y la práctica y para extraer nuevas generalizaciones a partir de la complejidad de las acciones; para ver las relaciones industriales a partir de diversas perspectivas y no de una sola; para rechazar las anteojeras de la ideología» (1978: 132-133).

En ese tiempo, el funcionalismo es una de las corrientes principales en el estudio de lo que acontece en el mundo de la industria, al menos en el mundo anglosajón. En 1958 se publica Sistemas de relaciones industriales, un texto importante en la constitución de este ámbito de estudio que se expande de manera acelerada en los años sesenta en un entorno dominado por la tradición del laissez faire, por tanto, de fuerte inhibición estatal en el desarrollo de las relaciones laborales.

Este proceso de expansión del nuevo ámbito de estudio no es extraño al contexto en el que se vienen articulando unas aproximaciones analíticas a partir de la emergencia de una serie de preocupaciones de matriz reformista en Estados Unidos desde principios de los años veinte y que ya se habían prodigado en el Reino Unido desde finales del siglo XIX. Tampoco es ajeno al contexto en el que se llevan a cabo ciertas investigaciones sociales de la realidad industrial; en este sentido, una referencia obligada es el movimiento de Relaciones Humanas, cuya perspectiva resulta compatible con el enfoque funcionalista que domina la sociología en los años cincuenta. Lo mismo va a suceder con la perspectiva analítica de las relaciones industriales que se despliega en ese tiempo y que, en este caso, responde a determinadas formas de racionalizar las necesidades de la acción política.

Por ello, uno de los objetivos del presente artículo es evidenciar los fundamentos bajo los que descansa esa voluntad de tratar intelectualmente las relaciones entre el capital y el trabajo, así como los de aquéllas otras que coinciden en el tiempo, examinando las distintas secuencias discursivas para extraer, al mismo tiempo, lo que aportan y las limitaciones que contienen. Esto supone repasar el estatus de las perspectivas sistémicas, hegemónicas en ese espacio y en ese tiempo, pero también otras como la de la teoría de juegos en la que se formaliza las interacciones entre los actores, así como los equilibrios que resultan para elaborar modelos microsociales de negociación y de resolución de conflictos; o la marxista, en la que las miradas se dirigen hacia determinados aspectos o problemas de las relaciones entre capital y trabajo, el papel del conflicto laboral, el control...

El fin que subyace a esta revisión parcial es poner de manifiesto cómo se configuran las relaciones laborales como dominio autónomo en un espacio geográfico, el del mundo anglosajón, y en un periodo determinado, el que se prolonga desde finales de la Segunda Guerra Mundial hasta la crisis de los setenta. Fuera quedan otros retazos de este periplo intelectual como el que 
tejen Mallet y Touraine, en Francia, centrado en la contribución sindical a la regulación del orden social en un entorno que registra importantes cambios tecnológicos. Una historia intelectual que, por otro lado, no se puede separar del entorno político y económico en el que se formaliza la aproximación al hecho sindical y a las relaciones laborales.

El artículo se inicia reconstruyendo los antecedentes de este ámbito académico en el mundo anglosajón para después extenderse ampliamente en la revisión propiamente dicha. Finalmente, se efectúa un balance sintético del estado en que se encuentra en los años setenta, extrayendo aquellos constructos teóricos considerados con mayor capacidad explicativa pero que pueden ser al mismo tiempo operativizados, antes de entrar en una nueva fase, la de «añoranza», en la que se van a abandonar las preocupaciones analíticas por la negociación colectiva, por la historia sindical, etc., en los trabajos de relaciones industriales para pasar a interesarse por cuestiones como el paro, la discriminación, la precarización... Ahora bien, más que este abandono y emergencia de objetos de estudio, más que una mayor especialización de la sociología académica (sociología del empleo, del trabajo...), lo que caracteriza el nuevo tiempo es el predominio de la aproximación neoliberal a los problemas del mundo laboral. Esto supone una ruptura con los planteamientos discursivos imperantes en las décadas anteriores, en las de la «edad de oro».

\section{Los "primeros tiempos»: emergencia de una preocupación}

Si hay que citar padres fundadores de la disciplina, una referencia obligada son Sidney y Beatrice Webb. Su sentido pragmático les inclina a la investigación empírica de la que han dejado testimonio en numerosos estudios sobre las instituciones (sindicatos, cooperativas, administraciones) de una Inglaterra en pleno apogeo económico. Por razones políticas, las que merecen una atención preferente son los sindicatos considerados como un medio de protección frente a los perjuicios de la competencia económica. Tres son los dispositivos que poseen para impulsar el proceso de democratización en el sistema productivo: los seguros mutuos, que cubren los riesgos (accidentes, paro...) a los trabajadores, pero no les garantizan ningún control de las condiciones de empleo y de trabajo; los convenios colectivos, que satisfacen esta laguna aunque no alcanzan al conjunto de los trabajadores, y la regulación legal, a la que corresponde asegurar la generalización de las reglas.

Aunque la aportación de los Webb con clara pretensión científica es importante, hay que dirigir la mirada hacia Estados Unidos para ver constituirse las relaciones laborales como disciplina, es decir, con un objeto de enseñanza e investigación delimitado en un contexto científico de carácter social a lo largo de buena parte del siglo XX. En ese lado del Atlántico, los efectos de la industrialización también se dejan sentir en el desarrollo de las relaciones laborales suscitando numerosas reflexiones entre los científicos sociales. Entre las más fecundas, las de John Rogers Commons y Selig Perlman, profesores de la Universidad de Wisconsin. El primero, influido por los Webb, centra su aten- 
ción en las reglas con el fin de mostrar cómo la práctica obrera y la acción colectiva desembocan en la producción de normas para regular las actividades laborales y estabilizar los conflictos de intereses. Asimismo, desarrolla una reflexión institucionalista que atiende en toda su importancia a lo colectivo y a la organización jerárquica. El segundo, Perlman, al igual que sus predecesores, está atento al peso de las prácticas y de las reglas que organizan la vida y las relaciones de trabajo, si bien su esfuerzo principal se dirige a explicar el hecho sindical: su contribución a transformar la mentalidad individualista de los trabajadores americanos y a promover la solidaridad entre ellos, en tanto que los empleos son escasos.

Pronto se sumaran otros estudiosos de la Universidad de Baltimore, que atribuyen un papel importante a las fuerzas exteriores del mercado en la explicación de la distribución del trabajo y de su precio y que, por ello, recibieron el nombre de «institucionalistas». Su concepción arraiga metodológicamente en la historia, la sociología y el derecho más que en la teoría económica que apenas aporta nada a la comprehensión de los problemas laborales. A este respecto, centran su atención en los sindicatos y la negociación colectiva, el derecho laboral o los seguros sociales. Así pues, una serie de rasgos caracterizan estos primeros trabajos: por un lado, una revuelta contra la esterilidad de la teoría económica clásica para tratar problemas del trabajo y, por otro, una voluntad reformadora que se esfuerza en legitimar el sindicalismo y proponer algunas reformas sociales, pero también el deseo de construir una teoría del movimiento sindical alternativa a la del marxismo.

A partir de la New Deal, pero sobre todo en los años posteriores a la Segunda Guerra Mundial, la nueva disciplina comienza a levantar el vuelo. Durante los casi veinte años siguientes, la investigación sobre «relaciones industriales»es frecuentemente descriptiva y, a menudo, histórica. En este sentido, la más singular es la que protagonizaron algunos jóvenes economistas de la Universidad de California, que abordan, con la panoplia de instrumentos propios de su profesión, problemas del mundo del trabajo: la movilidad y el proceso de búsqueda del empleo, las políticas sindicales, el impacto de la negociación colectiva, los factores que afectan a la estructura interna de los salarios y contribuyen a la emergencia de los mercados internos...

Así, el joven Dunlop elabora, en 1944, una aproximación «económica» al fenómeno sindical considerando los sindicatos como actores que aspiran a maximizar la renta de sus afiliados. Ross propone, en 1948, una aproximación "política»: cada líder sindical se preocupa por mantener el poder de su organización y se esfuerza por obtener aumentos salariales semejantes a los obtenidos en sectores próximos. Chamberlain elabora, en 1951, la teoría de la negociación colectiva concebida como medio de contrato salarial, forma de gobierno industrial, modalidad de management (Caire, 1991: 378). En este período, la negociación colectiva es vista como un instrumento destinado a mantener la paz social y el equilibrio de fuerzas del mercado.

En este contexto, se lleva a cabo, poco tiempo después, en 1960, el estudio comparativo de Kerr, Dunlop, Harbison y Myers, El industrialismo y el 
hombre industrial, en el que, entre otros aspectos, se aborda el impacto de la industrialización en los trabajadores y su reacción, sobre la base de la conceptualización que había efectuado Dunlop dos años antes en El sistema de relaciones industriales.

Estas perspectivas volcadas en los sindicatos y en las formas de producir reglas en las relaciones sociales en la industria, encuentran correlatos en otras partes del planeta a partir de mediados de los años cincuenta. Especialmente en Gran Bretaña, si bien, en este caso, la Escuela de Oxford está más abierta a los sociólogos, más receptiva a los presupuestos marxistas.

\section{El contexto intelectual}

Alejados de las inquietudes de quienes dan los primeros pasos en la conformación de las relaciones laborales como ámbito de reflexión sociológica, ciertos autores promueven y efectúan una serie de investigaciones sobre un aspecto singular de la realidad industrial: la organización. Se trata de investigaciones que no sólo apuntan los cambios registrados en el campo de la sociología industrial previo y posterior a la Segunda Guerra Mundial, sino que también configuran un contexto de referencia para quienes manifiestan preocupaciones por las «relaciones industriales». A ese respecto, es obligado citar el impacto que tuvieron algunos de esos análisis como los llevados a cabo por Roethlisberger y Dickson (1939) en la planta Hawtorne de Chicago de la Compañía Eléctrica Occidental o por Warner y Low (1947), en la industria de zapatos de Nueva Inglaterra, ambos efectuados dentro de lo que se denomina «Movimiento de las Relaciones Humanas», en los que se evidencia la influencia de los factores sociales en el comportamiento laboral de la mano de obra. Desde la perspectiva de Elton Mayo, su principal difusor, las soluciones a los problemas de desintegración social y de elevada conflictividad que acompañan a la quiebra de la sociedad preindustrial pasan por proporcionar a los individuos la posibilidad de pertenecer a pequeños grupos dentro del centro de trabajo unidos por una finalidad común que no encontrarán en el mundo exterior.

A pesar de que coexisten diferentes puntos de vista dentro del Movimiento de Relaciones Humanas, comparten una serie de supuestos que van a influir en el contenido y la forma de la investigación sociológica de la industria que tiene lugar en el mundo anglosajón. A este respecto y como ya es conocido, los trabajadores se perciben como algo más que simples «hombres económicos»: tienen necesidades sociales y no sólo económicas, de modo que su comportamiento se ve también afectado por factores sociales. Concretamente, se ven influidos por los pequeños grupos a los que pertenecen y con los que se identifican; así como por las formas de ejercer la dirección. Por tanto, se hace necesario abordar y entender la organización «informal» para comprender lo que acontece realmente en la industria. En coherencia con ello, la investigación en relaciones humanas se orienta hacia la exploración de las relaciones que tienen lugar en los pequeños grupos, el «liderazgo», y/o las oportunidades de participar personalmente en actividades de grupo. 
En esta perspectiva, el entorno económico e institucional en el que tienen lugar tales relaciones se omite, así como las condiciones de mercado en las que empresarios y gerentes adoptan sus decisiones. Dicha omisión facilita la asunción de que no existen conflictos intrínsecos de interés dentro de la empresa y la consideración de que, cuando se producen, son debidos a comunicaciones defectuosas, un mal liderazgo, incapacidad personal o fallos entre los miembros de la organización.

El enfoque de las «relaciones humanas» en los estudios de la industria y del trabajo resulta compatible con el análisis funcionalista que domina la sociología de los años cuarenta y cincuenta. La influencia funcionalista se aprecia en las referencias que Roethilisberger y Dickson hacen en su trabajo a Durkheim, Pareto o a Malinowsky y Radcliffe-Brown. Una influencia que se observa asimismo en la investigación sociológica británica dada su familiaridad con la sociología industrial americana ${ }^{2}$.

Pronto será replicado el enfoque de las Relaciones Humanas y el funcionalismo normativo, tanto en Estados Unidos como en Gran Bretaña, si bien hay que señalar que en este último Estado resulta más difícil asumir el conflicto industrial como patológico o que los sindicatos están equivocados. El entorno político británico es diferente y los sindicatos están más consolidados que en Estados Unidos dentro del sistema de relaciones laborales. En las primeras críticas, ya se hace referencia a la inclinación del movimiento hacia los círculos directivos, a su incapacidad para reconocer la racionalidad del comportamiento discrepante de los trabajadores y a su negación de los conflictos de interés económico (Watson, 1994: 45). Todo esto explica que fuera reconocida más fácilmente la legitimidad de los intereses de las partes en Gran Bretaña. De todas maneras, la perspectiva de las Relaciones Humanas no es la única fuente que inspira los planteamientos e ideas más generales de la sociología industrial británica. En su desarrollo, se observa la ascendencia de ciertas consideraciones de Marx, la clase y la conciencia de clase, y de Weber, la burocracia, como es perceptible en algunos textos que alcanzaron gran notoriedad en ese tiempo: el de Dennis, Henriques y Slaughter (1956) y el de Lockwood (1958).

Este proceso de crítica del funcionalismo normativo, así como de recuperación de clásicos para acercarse a la realidad social, se acentúa a finales de los cincuenta. Dahrendorf, entre otros, critica la perspectiva funcionalista y el modelo consensuado de sociedad a que conduce a partir del recurso a Marx y Weber en Las clases sociales y su conflicto en la sociedad industrial, lo que refleja el creciente interés sociológico por las perspectivas analíticas que se pueden derivar de los textos de Marx y Weber.

2. Sus planteamientos llegan asimismo al Estado español. A medida que el país se industrializa y la permisividad se acrecienta, comienzan a ser objeto de atención de algunos científicos sociales, más concretamente psicólogos como Siguán (1959) o Pinillos (1969), que elaboran textos divulgativos con comentarios más o menos críticos a las ideas defendidas por el publicista del pensamiento de la escuela de las relaciones humanas, Elton Mayo. 
Este pluralismo teórico cuaja poco tiempo después en tres perspectivas: funcionalismo, rama de la acción social o «interaccionismo» y teoría del conflicto, que van a evidenciar su forma de mirar en la sociología industrial de los años sesenta y setenta. Buena parte de los debates que tienen lugar en este tiempo se articulan alrededor de los problemas suscitados por estas tres perspectivas.

\section{El prisma funcionalista de Dunlop: los sistemas de relaciones industriales}

Sobre la base de que el mundo camina irreversiblemente hacia la industrialización - la tesis de la convergencia-, Dunlop arranca en el prefacio de su libro señalando que toda comunidad que se industrializa crea obreros y empresarios y que, entre unos y otros, se crea un entramado de relaciones que no es independiente de la acción del Estado. Es decir, reconoce que las sociedades industriales dan lugar a relaciones industriales, definidas "como el complejo de interrelaciones entre empresarios, obreros y organismos del gobierno». En este sentido, el objeto explícito del libro es proporcionar una teoría general de las relaciones industriales.

En ese entramado de relaciones industriales, establece tres áreas de experiencia: a) relaciones industriales dentro de una empresa, industria u otro segmento de un país, lo que permite comparaciones entre dichos ámbitos; $b$ ) relaciones industriales dentro del conjunto de un país que permiten comparaciones entre países, y $c$ ) relaciones industriales en el transcurso del desarrollo económico.

Así pues, Dunlop pretende analizar el funcionamiento de sistemas de relaciones industriales teniendo en cuenta que, independientemente de su tamaño, poseen ciertas propiedades y estructuras comunes, por un lado, y, por otro, que están sujetos y responden a determinadas influencias. A este respecto, considera que todo sistema de relaciones industriales está compuesto por tres clases de actores: los empresarios y sus organizaciones, los asalariados y sus representantes y los organismos gubernamentales, y da lugar a un complejo de reglas que tiene como fin gobernar el centro de trabajo y la comunidad laboral, ya que definen el estatus de los actores y regulan su conducta. Por tanto, coloca las normas y las reglas de las relaciones industriales en el centro del análisis e identifica los elementos del sistema que constituyen. Asimismo, supone que los actores de un sistema de relaciones industriales experimentan las presiones de un entorno en el que distingue tres contextos interrelacionados: el tecnológico (técnica utilizada en los puestos de trabajo), el económico-financiero (posición de las empresas en el mercado del producto) y el jurídico-político. Por último, el conjunto (sistema) se cimenta por una ideología, un conjunto de convicciones comunes que definen el rol, el lugar y la representación de los actores.

La noción de sistema de relaciones industriales es central en el discurso de Dunlop en tanto que es considerado un sistema analítico, «[...] en la misma esfera lógica que un sistema económico». Se trata de una consideración que se inscribe dentro de la tradición funcionalista, deudora de la descripción parso- 
niana de «sistema social», sobre la que superpone esquemáticamente, subsistema económico, subsistema de relaciones industriales. De aquí que considere que los instrumentos para explicar cada subsistema son específicos. Por otro lado, entiende que un sistema de relaciones industriales es una abstracción, como lo es un sistema económico - ninguno de los dos se ocupa del comportamiento total- . Por tanto, su descripción está limitada a ser una representación. En este entramado teórico, propone atender tres problemas particulares: el de las relaciones del sistema de relaciones industriales con el conjunto de la sociedad; el de sus relaciones con el subsistema económico, y el de la estructura propia del subsistema de relaciones industriales.

La referencia a la noción de sistema remite a la noción de función. En este sentido, asume la diferenciación analítica establecida por Parsons y la adapta al campo de las relaciones industriales. Cuatro son las funciones del sistema. Primera, la función adaptativa, que alude a los procesos de regulación y confección de reglas que «relacionan los actores con el entorno tecnológico y de mercado». Segunda, el imperativo de los fines que implica que la política del subsistema persigue la supervivencia y estabilidad del sistema de relaciones industriales, así como de la jerarquía de determinados actores necesarias para la consecución de los fines. Tercera, la de integración, que hace referencia al amplio campo de «entendimientos compartidos» y a la ideología común del sistema que relaciona los papeles individuales a las jerarquías y, a su vez, a las jerarquías entre sí. Y cuarta, la de mantenimiento del modelo y control de la tensión que es desempeñada por el papel del experto o profesional en los tres grupos de actores del sistema.

En este contexto, el sistema de relaciones industriales tiene unas determinadas relaciones con el sistema social. Por un lado, mientras la función adaptativa de la sociedad aporta riqueza y renta al sistema, la de relaciones industriales aporta reglas que incluyen los índices salariales y otras formas de compensación. Por otro, las funciones integradoras de ambos sistemas interaccionan mutuamente en una sociedad industrial.

Por tanto, aunque Dunlop no asume plenamente el discurso de Parsons, sin embargo, resulta evidente que sí extrae una serie de orientaciones, formalismo, conservadurismo, que resultan apropiados para la racionalización y legitimación de un ámbito de estudio particular que se va a desarrollar aceleradamente a partir de entonces. Las interacciones entre una y otra perspectiva fueron muy bien expuestas por Hyman a mediados de los setenta:

El análisis sistémico ofrece una alternativa ideológicamente aceptable a los que adoptan sus perspectivas pragmáticas, pero rechazan su lenguaje y sus conceptos poco sofisticados. Los imperativos de eficacia, de adaptación a las necesidades y al cambio, de reforma en el orden de las relaciones laborales pueden ser tratadas en una u otra perspectiva. Las principales preocupaciones de la sociología de Parsons son paralelas a las de los industrialistas ${ }^{3}$. 
Ahora bien, el discurso teórico de Dunlop no sólo abre el camino a la expansión de un ámbito de estudio, sino que supone también una inflexión significativa respecto al objeto de estudio y a los planteamientos existentes en ese tiempo en el examen de la realidad industrial. En primer lugar, su énfasis en considerar las normas que regulan el centro de trabajo y la comunidad laboral como el núcleo substantivo de las relaciones laborales en la industria no sólo es difícilmente rebatible, en tanto que normas de distintos tipos impregnan el mundo del empleo y del trabajo, sino que también suponen un distanciamiento respecto a lo que constituye el foco analítico de dos autores de referencia obligada, Taylor y Mayo, que centran su atención en los factores que aumentan el rendimiento laboral de los trabajadores. A este respecto, los planteamientos de Dunlop amplían la mirada en un sentido más sociológico (y jurídico). En segundo lugar, porque la consideración de las limitaciones de carácter técnico, económico y político que impone el entorno implica una ruptura en la concepción de la empresa como unidad cerrada válida en sí misma para su observación. En tercer lugar, frente a una visión muy restrictiva de la organización económica como suma de individuos que tienen intereses individuales diversos, Dunlop los reconoce junto a las organizaciones que los agrupan y defienden como intereses grupales diferenciados, con lo que da entrada a las asociaciones empresariales y a los sindicatos. Esta consideración supone un afianzamiento de la mirada sociológica. Y, por último, tanto en Sistemas de relaciones industriales, como en la obra colectiva realizada con Kerr, Harbison y Myers, Dunlop plantea la importancia de los estudios comparados con el fin de superar el aislacionismo de los análisis norteamericanos.

Resulta evidente que los planteamientos de Dunlop suponen un importante impulso en el estudio de las relaciones laborales. Lo reconoce alguien muy alejado de los presupuestos de Dunlop, como Hyman:

[...] estos análisis [...] son de gran importancia por su condición de intentos iniciadores de dar unidad teórica y precisión al estudio de las relaciones industriales; y por esta misma razón han resultado sumamente influyentes (1981: 21).

Entre otras razones porque como se acaba de escribir, normas de distinto tipo regulan el mundo del empleo y del trabajo. Ahora bien, definir el objeto de un ámbito de estudio en términos de normas es todavía restrictivo, a pesar de la ampliación y diversificación que encierra con respecto a miradas precedentes o coetáneas. Es lo que señalan otros autores británicos en congruencia con la experiencia británica. Pero además, en esa concepción del objeto de estudio subyace una determinada valoración: que el fin de las relaciones industriales es conseguir la estabilidad económica. En este sentido, la atención se centra en cómo contener y controlar los conflictos, más que en los procesos que los generan.

Asimismo, Dunlop reconoce que existen diferentes ámbitos normativos, el centro de trabajo, la empresa, rama de actividad económica, territorio, nación... Ahora bien, no explica como se relacionan unos con otros o si los 
procesos internos son iguales en todos ellos; tampoco explica si los actores se comportan de igual modo, sea cual sea el marco de intervención. Dunlop vuelve a caer como sus predecesores en una concepción cerrada del sistema, que implica a su vez una concepción muy estática del mismo en que sólo cambian los elementos internos al sistema. De aquí se infiere otra laguna en su argumentación teórica: la falta de perspectiva histórica; o mejor, el desplazamiento de su centralidad analítica a favor de un tratamiento y análisis de las relaciones laborales en un momento determinado (Estivill, 1978: 13).

$\mathrm{Y}$ es que la concepción de Dunlop del sistema de relaciones industriales se efectúa desde su mirada sobre la sociedad estadounidense, la sociedad más industrializada. Pero esta mirada no sólo es americanocéntrica sino también conservadora cuando en esa concepción subyace una apuesta porque los procesos implicados tengan lugar en el sentido de mantener la estabilidad y el equilibrio, porque las instituciones y procedimientos sean compatibles y porque el conflicto se autorresuelva. Esta perspectiva resulta más evidente cuando Dunlop considera que la ideología de los actores del sistema constituye una fuente de orden:

Un sistema de relaciones industriales crea una ideología o un cuerpo de ideas y creencias comúnmente compartidas, relativas a la interacción y papeles de los actores, que contribuye a mantener unido al sistema (353).

Por otro lado, los supuestos ideológicos que inspiran Sistema de Relaciones Indutriales, se hacen patentes no solo en los temas en que Dunlop vuelca su atención o en la forma de hacerlo, sino también en los aspectos que omite, la acción humana en tanto que motor de las relaciones sociales de la industria, y sobre todo, en las preguntas que elude contestar: una de las principales es la influencia de la estructura de propiedad y control vigente en las relaciones industriales; o más concretamente, si puede ser considerada como fuente de conflictos. Dunlop evade la respuesta aludiendo que dicha estructura es externa al estudio de las relaciones industriales que únicamente deben preocuparse de cómo los empresarios y sus asociaciones, los trabajadores y sus sindicatos y otras instituciones afrontan tales conflictos mediante reglas.

A pesar de todas estas limitaciones que hacen difícilmente aceptable la pretensión de Dunlop de que constituya una teoría general, Sistemas de relaciones industriales desencadena toda una serie de trabajos (Flanders, 1970 —. o. 1965-; Derber, 1969; Craig, 1975) en los años siguientes que tienen como objeto las "relaciones industriales», aunque no coinciden a la hora de delimitar lo que debe constituir el núcleo central de la teoría: la democracia industrial, según Derber; los términos y condiciones de empleo, según Craig, o las instituciones de regulación del trabajo, según Flanders y Clegg, que ya antes de la publicación del libro de Dunlop, y en contraposición abierta con la Escuela de Relaciones Humanas, señalaban que:

[...] somos conscientes de que centrar nuestra atención en las instituciones formales de las relaciones industriales puede suscitar las críticas de los que están 
influidos por las enseñanzas de la nueva escuela de «relaciones humanas en la industria» [...] [que] está en una fase temprana de su evolución y todavía tiene que proporcionar material que pudiera utilizarse para la enseñanza. [...] la mayor parte de [sus trabajos] publicados muestran una carencia deplorable de rigor histórico y, a veces, fallan al apreciar la naturaleza de la situación estudiada debido a la ignorancia de las instituciones formales que la rodean. Así pues, el estudio de las instituciones nos parece el paso preliminar apropiado a la hora de utilizar métodos ambiciosos (1954: V-VI).

\section{La perspectiva pluralista de la Escuela de Oxford: las instituciones de regulación laboral}

Las instituciones formales constituyen, pues, el objeto de estudio privilegiado por lo que se ha dado en denominar la "perspectiva pluralista de la escuela de Oxford». Una aproximación que ha sido considerada frecuentemente como la variante británica del modelo sistémico. A partir de los años sesenta, se registran cambios importantes en los temas que preocupan a quienes estudian la realidad industrial en el Reino Unido como consecuencia no sólo de la expansión y el mayor respeto que merecen la enseñanza y la investigación de las ciencias sociales, sino también de la influencia de la Comisión Donovan (Comisión Real de Sindicatos y Asociaciones Empresariales) que funciona activamente entre 1965 y 1968. En este último año, la Comisión reconoce la existencia de un sistema «formal» de negociación colectiva de la gran industria materializado en las instituciones oficiales y el sistema «informal» de negociación del trabajo a destajo, de complementos del salario mínimo y de realización de horas extras a nivel de fábrica, que queda en gran medida fuera del control de las asociaciones de empresarios y de los sindicatos. También reconoce que la evolución del sistema «informal» en un entorno de pleno empleo conduce a un aumento de la conflictividad y de la protesta laboral, huelgas no oficiales... La Comisión encuentra la solución a estos problemas en las relaciones laborales, en la intervención de partidos y sindicatos, con la colaboración del Estado. A través de dicha intervención, se deberían llevar a cabo reformas constitucionales para establecer procedimientos ordenados y eficaces de negociación colectiva de los salarios, así como un control bilateral de los mismos y de las prácticas laborales.

Se genera, así, un ambiente favorable para el desarrollo de la nueva disciplina que lleva a la construcción de un discurso teórico propio y a la investigación directa de las relaciones entre capital y trabajo. A este nuevo clima no son ajenos los planteamientos de Allan Flanders, que, en 1965, publica un folleto, Las relaciones industriales, ¿qué es lo que va mal del sistema?, reeditado en 1970, en el que no sólo aborda los problemas habituales de las «relaciones industriales británicas» (y a los que se ha hecho referencia), sino que también ofrece argumentos para considerar las relaciones laborales como un ámbito de estudio singular:

[...] su desarrollo debe apoyarse en el soporte mutuo de la teoría y la investigación [...] El inconveniente de fiarse de la teoría de cualquiera de las variadas disciplinas que abordan las relaciones industriales es que nunca pretenden ofre- 
cer un punto de vista integrado de todas las instituciones en este ámbito. En términos teóricos, estas disciplinas despedazan la materia al concentrar la atención en alguno de sus aspectos hasta la exclusión o el olvido comparativo de otros y una visión parcial de cualquier cosa, aunque sea acertada dentro de sus límites [...], siempre está necesariamente distorsionada y, por tanto, el significado de la noción de sistema de relaciones industriales (1970: 85).

Flanders aborda el contenido de las relaciones industriales tomando como referencia el libro de Dunlop y su consideración de que:

un sistema de relaciones industriales [...] es un sistema de normas que surgen bajo distintas formas: en la legislación y en los estatutos laborales, en las regulaciones sindicales, en los convenios colectivos y resoluciones de arbitraje de la autoridad laboral, en las convenciones sociales, en las decisiones de la dirección y en la "costumbre y la práctica aceptadas». Esta lista no es de ningún modo exhaustiva, pero «normas» es la única descripción genérica que puede utilizarse para referirse a estos variados instrumentos de regulación. En otras palabras, esta materia se ocupa de las relaciones reguladas o institucionalizadas en el interior de la industria. [...] El estudio de las relaciones industriales puede, por consiguiente, describirse como un estudio de las instituciones de regulación del trabajo (1970: 86).

Comparte, asimismo, la preocupación de Dunlop por la estabilidad de las relaciones industriales estando igualmente convencido de que los conflictos de intereses pueden ser resueltos por medio de la negociación y, en el peor de los casos, pueden ser mantenidos dentro de límites socialmente soportables. Ahora bien, se diferencia de Dunlop en que renuncia a la concepción integradora de la ideología, ya que reconoce la pluralidad de intereses y la diversidad de puntos de vista de los actores. Esto explica que esté preocupado por los mecanismos de producción de reglas en tanto que instituciones en las que organizaciones que representan intereses diferentes se enfrentan y negocian lo que les lleva asimismo a ahondar críticamente en los planteamientos de los Webb. En primer lugar, destaca la importancia de las fuentes extraeconómicas en la negociación colectiva. Contrariamente a lo que pensaban los Webb, las negociaciones no son reducibles a relaciones o tratos de mercado. O, dicho de otra manera, no deben ser aprehendidas únicamente a través de la lógica económica, ya que la producción de reglas resulta asimismo de la confrontación de organizaciones que poseen ideologías diferentes y que incluso tienen que administrar intereses heterogéneos en su interior. La negociación colectiva remite así, también, a otras lógicas, la institucional, la política.

En segundo lugar, la negociación colectiva es considerada en un sentido amplio, de modo que no puede ser limitada a la elaboración de reglas, ya que existe una interacción entre este proceso y su aplicación. Además, junto a los acuerdos que regulan las condiciones de empleo y trabajo, los actores de la negociación colectiva negocian asimismo acuerdos de procedimiento para regular sus propias relaciones. Estas reglas de procedimiento gobiernan, entre otras 
cosas, los conflictos y el recurso al arbitraje. Todos estos planteamientos sobre la negociación son considerados válidos para las sociedades pluralistas en las que la regulación de las relaciones laborales se basa en la libertad de contratación y de asociación y en las que los conflictos sociales son legítimos.

Flanders concentra, pues, su atención en la negociación colectiva considerada el dispositivo básico de producción de reglas y de regulación de conflictos. En el contexto descrito, se entiende que las «relaciones industriales» deben estar dedicadas al estudio de las «instituciones de regulación laboral», lo que está relativamente próximo a la preocupación de Dunlop, los «sistemas de normas» que producen empresarios y sindicatos en su interacción, aunque es perceptible un esfuerzo de Flanders por dar cabida no sólo a las estructuras sistémicas, sino también a los procesos de relaciones industriales, no sólo a las variables de naturaleza estructural, sino también a las de comportamiento, lo que le acerca de alguna forma (y en alguna medida) a la perspectiva behaviorista de la teoría de juegos. Ahora bien, dicha concepción plantea algunos problemas, el principal, que descentra la mirada de las estructuras reales de poder y de la dinámica económica y tecnológica de la sociedad, y junto a esta desviación de la atención del poder, otro problema, la pérdida de centralidad del conflicto y de la inestabilidad en los procesos de relaciones industriales en beneficio de una determinada concepción de la regulación.

Esa parte de la argumentación de Flanders, la relativa al poder, es la que resulta más controvertida. Considerado en términos excesivamente legalistas, institucionalistas, se deriva una igualdad formal de las organizaciones que representan los intereses de las partes, en tanto que poseen estructuras burocráticas, derecho a negociar..., lo que confiere una legitimidad, aceptación y justicia a los resultados de la negociación. Ahora bien, que sean iguales formalmente (en términos legales) no quiere decir que tengan el mismo poder real, es decir, la misma capacidad de incidir políticamente en el desarrollo de las relaciones entre dirección y empleados, ni concreta ni estratégicamente.

Así pues, aunque Flanders reconoce que existen conflictos de intereses inherentes a las relaciones entre empresarios y trabajadores, sin embargo, sus planteamientos tienen una fuerte afinidad con un funcionalismo normativo de carácter pluralista, ya que asume que tales conflictos pueden estar regulados y contenidos dentro de una estructura institucional configurada normativamente, al mismo tiempo que no tiene en cuenta (o no concede suficiente importancia) a la irresolubilidad de los conflictos o a las desigualdades de poder que permiten a una parte imponer más fácilmente sus decisiones a la otra.

\section{Las perspectivas sistémicas a finales de los sesenta: primera crisis}

Se expande, pues, un nuevo ámbito de conocimiento: el de las «relaciones industriales». En esa expansión se dejan sentir de distintas maneras (y de modo desigual) las consideraciones que Coser había efectuado sobre las funciones del conflicto laboral poco tiempo antes; sobre todo, la de «institucionalización del conflicto». Dicha noción es uno de los fundamentos de buena parte 
de los trabajos en sociología de las relaciones laborales desde finales de los cincuenta. Y no sólo en los aludidos, sino también en otros como el diagnóstico de Kerr, Dunlop, Harbison y Myers en El industrialismo y el hombre industrial sobre el imparable desarrollo de un «industrialismo pluralista» en el que la protesta de los trabajadores contra el impacto del mismo «se organiza, se canaliza y se controla» (241), dando lugar a los sistemas de relaciones industriales. Uno de los presupuestos básicos de este tipo de análisis es que los conflictos de intereses entre trabajadores y empresarios, que desde entonces son claramente expresados por instituciones legítimas representativas, pueden ser fácilmente contenidos y desactivados.

Este movimiento, que tiene mucha fuerza en la tradición angloamericana, supone un peculiar desarrollo teórico de las «relaciones industriales». En él subyace un paradigma que comporta dos postulados fundamentales. El primero implica una concepción naturalista de los intereses: los actores básicos del «sistema de relaciones industriales» son una multiplicidad de individuos y de organizaciones que representan sus intereses; las colectividades más amplias no tienen reconocida su existencia más que a través de las alianzas y los sistemas de organizaciones articulados por (y sobre) estos actores básicos. El segundo - más claro en el caso de ciertos autores que en el de otros- refleja una visión americanocéntrica de la naturaleza y de los fines de los sindicatos: a partir de una visión restrictiva de los intereses legítimos de los trabajadores, se realiza una apología de la negociación colectiva y una definición de los sindicatos como organizaciones de los trabajadores que son por naturaleza economicistas, acomodadas y respetuosas con la jerarquía establecida.

El peso de estos postulados en las perspectivas que estudian las «relaciones industriales» se comprende cuando se los sitúa históricamente. A este respecto, lo primero que hay que tener en cuenta es el clima ideológico de la guerra fría que presiona a los universitarios para que desarrollen un cuadro de análisis explícitamente antimarxista. Este clima tiene repercusiones sobre el análisis sociológico en general y, por supuesto, en el de aspectos particulares, como las relaciones laborales. Lo segundo que hay que considerar es que el entorno en el que las relaciones laborales se instauran como materia académica es el del capitalismo en plena expansión de posguerra y de hegemonía del imperialismo americano. Se trata de una base favorable para una resolución «de suma positiva» de los conflictos entre capital y trabajo, especialmente en los países desarrollados. El modelo del «industrialismo pluralista» es el producto de la misma coyuntura que hace nacer construcciones del tipo «sociedad de la abundancia», el «fin de las ideologías», y el «sistema de convergencia social».

A finales de los sesenta, el entorno comienza a modificarse radicalmente. Recesión económica y reducción de los beneficios limitan el margen de las concesiones empresariales en las negociaciones de los salarios, al mismo tiempo que la subida de precios aumenta las exigencias de los trabajadores. Otras formas de racionalización empresarial en la gestión de mano de obra se activan. Frecuentemente, se pone el acento en controlar el hecho mismo del empleo, es decir, se hace hincapié en aquellas formas que son menos susceptibles de con- 
ducir a compromisos. Las iniciativas promovidas desde el Estado suponen un distanciamiento de las orientaciones mantenidas hasta entonces.

Se abre, así, un proceso de inestabilidad social y política como resultado de una mayor radicalidad de los objetivos y de las formas de acción obrera. Las teorías optimistas de los especialistas universitarios de posguerra en «relaciones industriales» que consideran factible controlar el conflicto laboral pierden influencia temporalmente. Al mismo tiempo, la relativa calma que se conoce en las relaciones internacionales se traduce en un cierto relajamiento de las presiones que había conocido la vida intelectual durante la guerra fría. En esta coyuntura, tanto en el análisis de las relaciones laborales como en el de las ciencias sociales en general, se constata una tendencia creciente a buscar fundamentos teóricos en unas aproximaciones más radicales, y más próximas al marxismo.

\section{El enfoque marxista: los procesos de control sobre las relaciones de empleo}

La mayor parte de estos trabajos sobre «relaciones industriales» son rechazados por el pensamiento crítico de ese tiempo. Una muestra ilustrativa es el comentario que merece la expresión misma «relaciones industriales»: "eufemismo consagrado para designar el conflicto permanente, unas veces agudo, otras atenuado, entre los capitalistas y los trabajadores» (Miliband, 1970: 79). La razón esgrimida es que ayudan al control ejercido por las clases dominantes. A esta primera acusación de servidumbre a las gerencias y a los capitalistas, determinados autores marxistas añaden más tarde una crítica dirigida a los fundamentos intelectuales de este dominio de estudio y consideran que no se puede comprender plenamente el proceso de "regulación del empleo y del trabajo» más que analizándolo en un contexto definido, por un lado, por la dinámica de la producción y de la acumulación capitalista —el entorno económicoy, por otro, por una concepción más general de las relaciones sociales y políticas - el entorno sociopolítico- (Hyman, 1979: 423). En este sentido, las actividades de empresarios y trabajadores deben ser analizadas a partir de conceptos como las relaciones de producción y la lucha de clases.

Ahora bien, aún rechazando la aproximación académica prototípica (y conservadora) a las "relaciones industriales», no se puede negar que la realidad empírica a la que se refiere tal vocablo tiene una importancia práctica y teórica considerable para cualquier tradición intelectual y, por supuesto, para la marxista. Y ello independientemente de que se considere relevante incluir dos postulados básicos en la problemática de las «relaciones industriales» y en su estudio. El primero es que las relaciones sociales de producción reflejan y reproducen una oposición estructurada de intereses entre capital y trabajo (por tanto, suponen un antagonismo estructurado). El segundo es que el capitalismo organiza al mismo tiempo los trabajadores sobre una base colectiva - la del proceso de producción - y genera en consecuencia la base material para una resistencia efectiva al capital y a los objetivos del modo de producción capitalista. 
Hubiera sido de esperar, pues, que los autores ubicados en la corriente marxista hiciesen esfuerzos importantes, no sólo para criticar, sino también para reinterpretar lo que es producido bajo el vocablo de «relaciones industriales» (Hyman, 1979: 424). Y, sin embargo, sorprenden los escasos esfuerzos que se dedicaron para impulsar, sistemáticamente en los años de posguerra, otro cuadro de análisis de esta realidad concreta que constituye el ámbito de estudio de los especialistas universitarios de las «relaciones industriales». Esto refleja la falta de bases sólidas para una teorización en este sentido en la tradición marxista. El entorno de los trabajos de Marx y Engels no era el de un sindicalismo tradicional, ni de una negociación colectiva institucionalizada; sus comentarios sobre estos temas son respuestas a problemas inmediatos más que un análisis de fondo. Lo que se puede esperar directamente de estos trabajos es, pues, limitado. En sus escritos no se encuentra las noción de "relaciones industriales». Tampoco se hallan referencias a la institucionalización de un modo de regulación de los conflictos de intereses a pesar de la atención que han prestado al mercado de trabajo, la empresa o los primeros sindicatos; y cuando se refieren a éstos, privilegian la lucha contra la explotación y la pauperización y olvidan las acciones a favor de una regulación de los salarios o de las condiciones laborales.

En los años posteriores a la Segunda Guerra Mundial, en la época de compromiso de clase, característica del periodo keynesiano de posguerra, tampoco se desarrolló una teoría de matriz marxista en ese sentido. Entre otras razones, porque se concentraron los esfuerzos en «eximir» a la clase obrera organizada (de la acusación) de ser un actor potencialmente revolucionario; en presentarla con un nuevo talante. Este contexto sirvió para promover una gran diversidad de reflexiones que, dentro de la corriente marxista, y a pesar de las divergencias de fondo, estaba de acuerdo en minimizar el significado de la organización y de la acción obrera en el mundo desarrollado. Algunas expresiones ilustrativas pueden ser, según Hyman, determinadas versiones del estructuralismo académico en donde la intervención humana es excluida; los alegatos a favor de los partidos obreros y de los grupos de acción; o los discursos que sitúan el motor de la revolución mundial en los grupos sociales «marginales» o en el Tercer Mundo. Estas reflexiones, explícitamente o no, aceptan uno de los argumentos centrales de los analistas tradicionales de las «relaciones industriales»: "las luchas, en los países industriales avanzados, se contendrían e institucionalizarían desde entonces» (1979: 425).

Habrá que esperar a principios de los setenta para que el contexto cambie. Por un lado, porque la institucionalización estable va obligando a replantear las aproximaciones iniciales a las "relaciones industriales», lo que entraña un cambio de perspectiva en algunos autores marxistas. Por otro, porque la conflictividad laboral no sólo se reactiva en ese tiempo, sino que cambia, asimismo, sus formas de producción y de expresión. En este ambiente, surgen nuevas tendencias analíticas que conceden importancia a la organización y a las luchas colectivas de los trabajadores, pero también a los procesos de mediación institucional en los conflictos de trabajo: un análisis que comienza a indagar sus características, su origen y sus contradicciones internas. 
Uno de los primeros análisis sistemáticos fue desarrollado por R. Hyman en su «introducción marxista» a las relaciones laborales en el año 1975 y publicado en castellano en 1981. En este libro adopta una posición claramente opuesta a la oferta teórica dominante en los sesenta, la formulada por Dunlop y Flanders. A estos autores opone una economía política de las relaciones laborales que cuestiona la definición estrecha y unívoca de las relaciones industriales: «definir su objeto exclusivamente en términos de normas es demasiado restrictivo y contiene desgraciadamente tonos valorativos. Implica que la tarea de las relaciones industriales es lograr el mantenimiento de la estabilidad y la normalidad en la industria. La atención se centra en cómo se contiene y controla un conflicto, más que en los procesos a través de los que se generan los desacuerdos y los conflictos» (Hyman, 1981: 21). Por tanto, que la consideración de la estructura de la propiedad y del control vigente en la empresa como fuente inevitable de conflicto se ignore en tanto que hecho externo al ámbito de estudio de las relaciones industriales que sólo se deben ocupar del modo en que empresarios, sindicatos y otras instituciones afrontan tales conflictos.

En el nuevo tratamiento que reciben las «relaciones industriales», el orden y la regulación no constituyen más que una parte de sus dimensiones. Si se tienen en cuenta los procesos contradictorios y los intereses antagonistas que dividen a los actores, entonces el conflicto y el desorden se convierten en objetos de estudio importantes. La preocupación de Hyman es redefinir el ámbito de estudio para incluir el conflicto industrial, sus causas, sus consecuencias..., es decir, "los procesos de control sobre las relaciones de trabajo» (1981: 22). Desde un punto de vista teórico, tales procesos no son comprehensibles ni explicables más que dentro de un contexto que incluya la estructura social, la dinámica de acumulación y de crisis, así como las correlaciones entre fuerzas sociales.

Estos planteamientos irrumpen en ese nuevo clima intelectual que se configura a partir de la ruptura de la relativa estabilidad del capitalismo de posguerra entre finales de los sesenta y principios de los setenta y que constituye un estímulo para la controversia dentro de la tradición marxista. Uno de los debates significativos se articula alrededor de la ley de la caída tendencial de la tasa de beneficio y su relación con el análisis de la dinámica cotidiana de la conflictividad social, lo que va abriendo paso al análisis de la realidad concreta, algo que había estado ausente en las décadas anteriores en la tradición marxista. Por una parte, la resistencia colectiva de los trabajadores es teorizada como fuente de crisis de la tasa de beneficio, por otra, y relacionada con la consideración anterior, las estrategias específicas de las gerencias hacia los trabajadores son tenidas en cuenta en tanto que implican un desarrollo desigual de modelos y de problemas en la acumulación de capital (Hyman, 1979: 427).

Este último hecho está en el origen de otro de los aspectos que merece atención en esta controversia abierta: el proceso de trabajo. Dos precisiones previas se imponen. Una primera que, a menudo, los defensores tradicionales de las «relaciones industriales» realizan análisis sofisticados de la «regulación de tareas» y de los «sistemas normativos de relaciones industriales», sin reconocer 
que los procedimientos e instituciones sobre los que concentran su atención encuentran sus fundamentos en la esfera de la producción. Una segunda, que el análisis efectuado por Marx del proceso de producción, especialmente, en lo relativo a la cuestión de las modalidades y consecuencias de las transformaciones de la fuerza de trabajo comprada por el capital en trabajo efectivo realizado por el asalariado, no es suficientemente atendido en la literatura marxista de posguerra.

A principios de los setenta, es objeto de estudio nuevamente con la publicación de la obra de Braverman, Trabajo y capital monopolista, y con el eco que adquiere. Resulta evidente que el carácter específico del proceso de trabajo capitalista, su función como motor de la acumulación y del valor, su dependencia con respecto a un sistema de control obligatoriamente coercitivo, proporcionan una base de elucidación de los problemas teóricos que las aproximaciones tradicionales de la sociología industrial apenas comienzan a formular. En primer lugar, el acento puesto sobre este proceso aclara el carácter concreto y la naturaleza ambivalente de lo que los especialistas de las relaciones industriales denominan «relaciones manageriales». En segundo lugar, indica el carácter de clase de la función managerial. En tercer lugar, reconoce que la dinámica inherente al modo de producción capitalista se refleja asimismo en las constantes crisis del proceso productivo que amenazan la estabilidad de las formas institucionalizadas de resolución de los conflictos. En definitiva, evidencia que las experiencias contemporáneas de «racionalización» empresarial han de ser situadas históricamente. Además, esta atención al proceso de trabajo provoca que la mirada se concentre también en los cambios profesionales con respecto a la producción, lo que conduce a otro aspecto importante en el análisis marxista de los setenta, la diferenciación interna de la clase obrera que se deja sentir en el desarrollo de algunos aspectos de las «relaciones industriales»: la organización colectiva de los trabajadores, los fines y estrategias que persiguen sus distintos tramos o segmentos o sus formas de acción colectiva. De este modo, los debates que tienen lugar ayudan a explorar las lagunas de la tradición marxista.

Así pues, los análisis de Hyman tienen el mérito de abrir las relaciones industriales a un ámbito histórico y social más amplio —al análisis genéticohistórico- y, en este sentido, permanece como una de las contribuciones más pertinentes del mundo anglosajón. Ahora bien, su economía política de las relaciones industriales no está centrada efectivamente en el objeto de estudio principal que se había delimitado; encierra un riesgo de que se difumine en una concepción demasiado global de las relaciones de producción capitalista. Aún así, su perspectiva contiene un potencial heurístico importante, ya que ayuda a comprender e interpretar la lógica de las combinaciones posibles entre un conjunto de variables determinantes cada vez más amplio.

En lo que se refiere al otro autor, Braverman, aunque no descuida el entorno de la empresa, sin embargo, su enfoque se centra más en el nivel micro. En este sentido, Braverman, al igual que los autores sensibles al control del proceso de trabajo que protagonizaron el debate sobre el proceso de trabajo, contri- 
buyen al progreso de las reflexiones teóricas relativas a la "política de trabajo» y a la «micropolítica» en la empresa.

\section{Teoría de juegos y behaviorismo: la negociación colectiva}

Las aproximaciones precedentes al ámbito de las «relaciones industriales» dirigen su interés prioritariamente a los programas de acción configurados por las estructuras organizacionales (o institucionales). Se interesan además por su lógica de evolución así como por sus resultados en términos de integración y de regulación sociales. La preocupación de las teorías de la acción no está próxima. En esta perspectiva, importan sobre todo las relaciones entre actores en sus comportamientos estratégicos. A diferencia de las perspectivas más deterministas (teoría sistémica, marxista), los textos que plantean este tipo de inquietudes analíticas privilegian el análisis de las relaciones políticas, de los juegos de poder..., en el seno de la empresa y de las organizaciones en general.

Uno de los primeros textos elaborados en este contexto es el de Tom Burns (1961-1962), que se interesa por la empresa y otras unidades de producción en tanto que campos de acción. Ni una ni otras son consideradas sistemas de organización programados y cooperativos, ni simples sistemas de dominación. Son conjuntos de juegos micropolíticos animados por actores dotados de poderes diferentes y capaces de desarrollar estrategias conflictivas. La política es identificada con el poder que adopta la forma de una relación de intercambio entre actores. A este respecto, el margen de libertad y de negociación de que dispone cada actor representa para sus adversarios una fuente de incertidumbre.

Precisamente, las negociaciones entre actores de las relaciones industriales constituyen el objeto de estudio de una de las primeras variantes de la teoría de la acción, la de Richard Walton y Robert McKersie, que desarrollan en pleno apogeo funcionalista, en 1965, una teoría behaviorista. Desde una perspectiva diferente a la de Dunlop, la de la teoría de juegos que permite formalizar las interacciones entre varios actores y determinar los equilibrios que pueden resultar, observan las relaciones formales para elaborar modelos microsociales de negociación y de resolución de conflictos. Tipifican cuatro situaciones ideales:

1. ${ }^{a}$ La negociación distributiva remite a aquellos entornos en que se producen conflictos puros de intereses, es decir, que se pueden formalizar recurriendo a la teoría de juegos, como una situación de juegos de suma nula, ya que lo que gana un protagonista, lo pierde el otro. Los conflictos salariales son una muestra ilustrativa.

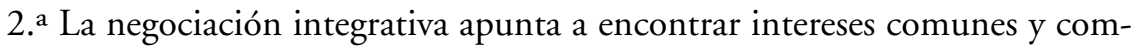
plementarios en la resolución de problemas que separan a las dos partes; es decir, remite a aquellas situaciones en las que las dos partes confluyen en un esfuerzo común para desembarcar en una solución satisfactoria para todos (juego de suma positiva). Por ejemplo, un acuerdo sobre enriquecimiento de tareas puede, al mismo tiempo, estimular la productividad y atenuar la penosidad del trabajo. 
3. ${ }^{a}$ La estructuración de las actitudes se refiere a una dimensión importante de la negociación que consiste en mantener o modificar el vínculo social que funda la interacción; por tanto, se refiere a la presión que una parte puede ejercer sobre la otra, con el fin de reducir su potencial de oposición o de aumentar su capacidad de cooperación. A este respecto, la gama de acciones es variada, va del conflicto abierto a la connivencia estrecha pasando por el compromiso neutro.

$4{ }^{a}$ La negociación intraorganizacional o interna remite al conjunto de deliberaciones en el interior de las organizaciones implicadas en la negociación cuyo fin es ajustar los intereses de los sectores representados por los negociadores con los resultados obtenidos, lo que no siempre es fácil, ya que las partes negociadoras están sometidas frecuentemente a una doble presión: la de su interlocutor, pero también la de los miembros de la organización que representan y a los que no es fácil hacer aceptar los términos del compromiso aprobado en su nombre.

Mediante datos empíricos, ambos autores muestran como a cada una de estas situaciones de negociación corresponden una serie de tácticas que pueden practicar los actores sociales para conseguir sus fines.

En esta perspectiva, es perceptible la influencia de la obra de Anselm Strauss y otros autores (1963), que ponen énfasis en estudiar los «órdenes» de corporaciones, universidades, hospitales..., como resultado de un proceso continuo de negociación y ajustes entre grupos. Con ello, el discurso se articula en torno a un eje situado en el otro extremo del espectro de enfoques ya analizados, que centran su atención en los aspectos estructurales de las organizaciones (o de instituciones), lo que supone una reorientación importante. En ese tipo de aproximaciones, se entiende que todos los órdenes sociales son negociados, porque son el producto de interacciones entre actores individuales y colectivos. Precisamente, este énfasis suele suponer descuidar las condiciones en las que tales acciones e interacciones tienen lugar. En una revisión crítica de las teorías del «orden negociado», Day y Day (1977) sugieren que tienen varias limitaciones. Entre otras, sus omisiones, por un lado, del medio exterior y, por otro, de los rasgos estructurales de una sociedad más amplia. De ahí que consideren que "la teoría tiene que tener una base política, estructural e histórica mayor de la que ha tenido en el pasado» (140). O, dicho de otra manera, el énfasis en el modo en que se negocia el «orden» debe ser complementado mediante el examen de las condiciones del entorno en el que tienen lugar tales procesos.

\section{Un balance de la «edad de oro»}

Resulta patente que en las casi dos décadas transcurridas desde que Dunlop desarrolla en 1958 un análisis de matriz sistémico, se registran importantes esfuerzos de sistematización de las «relaciones industriales», es decir, de delimitación de su objeto de estudio en torno al que se desarrollan conceptos, 
argumentos y métodos. Algunos de los más representativos del mundo anglosajón, el territorio en el que se han realizado por antonomasia, se han analizado poniendo de manifiesto su fundamentación teórica. A finales de los setenta existe, pues, un conjunto diversificado de interpretaciones de cuyos constructos y enfoques metodológicos se nutren posteriormente numerosos autores para intentar estudiar las «relaciones industriales» con una mirada frecuentemente ecléctica; sobre todo, debido a que el desarrollo de las instituciones no responde a un modelo único ni predeterminado.

Antes de hacer referencia a aquéllos que se consideran más significativos en la constitución del ámbito de estudio de las "relaciones industriales», hay que insistir en primer lugar que aunque las perspectivas analizadas no son necesariamente excluyentes, sin embargo, sus raíces, su alcance y su metodología no son similares y, en segundo lugar, y en relación con lo anterior, que el potencial heurístico de cada una de ellas varía más explícitamente según el interrogante y/o el aspecto del objeto de estudio privilegiados: la eficacia comparada de los sistemas de relaciones industriales, las instituciones de regulación laboral, el conflicto y el control de las relaciones laborales, la negociación, etc.

Pues bien, a su alrededor, son perceptibles una serie de constructos, antagonismo estructurado, orden negociado, que permiten una aproximación fructífera al desarrollo de la mediación institucional centrada en los actores y, más concretamente, a los cambios institucionales que se producen, a las condiciones que los posibilitan e incluso vaticinar su orientación más probable. Pero, también, es observable la operatividad del método genético-histórico en el estudio de las «relaciones industriales». A través de la reconstrucción racional del proceso en el que emergen organizaciones e instituciones se puede ver la dinámica social que promueven determinando la (re)constitución de actores cuyas acciones estratégicas e interesadas contribuyen a su vez a la (re)creación de nuevas instituciones. Esta creación conjunta no está ni planificada ni estructuralmente determinada, sino que resulta de interacciones múltiples y de un proceso de socialización que modela igualmente a los actores. En este sentido, se evidencia de qué maneras sus estrategias e interacciones están condicionadas. Con estos elementos de la construcción teórica, se pueden explicar de manera satisfactoria a la vez el conflicto y el consenso y dar cuenta, asimismo, de la evolución del desarrollo institucional que acompaña a las relaciones de empleo.

\section{Bibliografía}

Braverman, H. (1978). Trabajo y capital monopolista. México: Nuestro Tiempo. Burns, T. (1961-1962). "Micropolitics. Mechanism of institutional change». Administrative Science Quaterly, 6, p. 257-281.

CAIRE, G. (1991). «Des relations industrielles comme objet théorique». Sociologie du Travail, n. ${ }^{\circ} 3$, p. 375-401.

Coser, L.A. (1961). Las funciones del conflicto social. México: Fondo de Cultura Económica (e. o. 1956). 
CRAIG, A.W. J. (1975). «A framework for the analysis of industrial relations systems». En BARRETT, B. (ed.). Industrial relations and the wider society. Collier MacMillan, p. 8-26.

DAHRENDORF, R. (1979). Las clases sociales y su conflicto en la sociedad industrial. Madrid: Rialp (e. o. 1957).

DAY, R.; DAY, J.V. (1977). «A review of the current state of negotiated order theory: an appreciation and a critique». The sociological Quartely, n. ${ }^{\circ}$ 18, invierno, p. 126-142.

Dennis, N.; Henriques, F.; Slaughter, C. (1956). Coal is our life. Londres: Eyre \& Spottiswoode.

DERBER, M. (1969). "Industrial democracy as an organizing concept for a teheory of industrial relations». En SOMERS, G. Essays in industrial relations theory. Iowa State University Press.

Dunlop, J.T. (1978). Sistemas de relaciones industriales. Barcelona: Península (e. o. 1958).

Estivill, J. (1978). «Prólogo». En Dunlop, J.T. (1978). Sistemas de relaciones industriales. Barcelona: Península (e. o. 1958).

Flanders, A. (1970). Management and Unions. The theory and reform of industrial relations. Londres: Faber\&Faber (e. o. 1965).

Flanders, A.; ClegG, H.A. (eds.) (1954). The system of industrial relations in Great Britain. Oxford: Blackwell.

HyMan, R. (1979). «La théorie des relations industrielles: une analyse matérialiste». Sociologie du Travail, n. ${ }^{\circ}$ 4, p. 418-438.

- (1981). Relaciones industriales. Una introducción marxista. Madrid: Blume (e. o. 1975).

KeRR, C. (1978). «Industrial relations research: a personal retrospetive». Industrial relations, mayo, p. 131-142.

Kerr, C.; Dunlop, J.; Harbison, F.; Myers, Ch. (1963). El industrialismo y el hombre industrial. Buenos Aires: Eudeba (e. o. 1960).

LOCKWOOD, D. (1959). The blackcoated worker. Londres: Allen\&Unwin.

Miliband, R. (1970). El Estado en la sociedad capitalista. México: Siglo XXI.

PINILLOS, J.L. (1969). Las ciencias humanas y la organización industrial: nuevos caminos. Madrid: Instituto Superior de Dirección de Empresa.

Roethlisberger, F.J.; DicksOn, W. J. (1964). Management and the worker. Nueva York: Wiley, (e. o. 1939).

Siguan, M. (1959). Problemas humanos del trabajo industrial. Madrid: Rialp.

Strauss, A.; Schatzmann, L.; Ehrlich, D.; Bucher, R.; Sabschin, M. (1971). "The hospital and its negociated order». En CASTles, F.G., MurRaY, D.J.; PORTER, D.C. (eds.). Decisions, organizations and society. Harmondsworth, Midds: Penguin, p. 103-123 (e. o. 1963, en FrIEDSON, E. (ed.). The hospital in the modern society. Nueva York: Macmillan, p. 147-169.

WARNER, W.L.; LOW, J.O. (1947). The social system of the modern factory. New Haven, Conn.: Yale University Press.

WALTON, R.E.; MCKERSIE, R.B. (1965). A behavioral theory of labor negociations. An analysis of a social interaction system. Nueva York: ILR Press, Ithaca [2a ed. 1991.]

WaTSON, T. (1994). Trabajo y sociedad. Barcelona: Hacer. 